\title{
Impact of epinephrine and norepinephrine on two dynamic indices in a porcine hemorrhagic shock model
}

\author{
Raphaël Giraud, MD, MSc, Nils Siegenthaler, MD, Diego Arroyo, MD, \\ and Karim Bendjelid, MD, PhD, Geneva, Switzerland
}

BACKGROUND: Pulse pressure variations (PPVs) and stroke volume variations (SVVs) are dynamic indices for predicting fluid responsiveness in intensive care unit patients. These hemodynamic markers underscore Frank-Starling law by which volume expansion increases cardiac output (CO). The aim of the present study was to evaluate the impact of the administration of catecholamines on PPV, SVV, and inferior vena cava flow (IVCF).

METHODS: In this prospective, physiologic, animal study, hemodynamic parameters were measured in deeply sedated and mechanically ventilated pigs. Systemic hemodynamic and pressure-volume loops obtained by inferior vena cava occlusion were recorded. Measurements were collected during two conditions, that is, normovolemia and hypovolemia, generated by blood removal to obtain a mean arterial pressure value lower than $60 \mathrm{~mm} \mathrm{Hg}$. At each condition, CO, IVCF, SVV, and PPV were assessed by catheters and flow meters. Data were compared between the conditions normovolemia and hypovolemia before and after intravenous administrations of norepinephrine and epinephrine using a nonparametric Wilcoxon test.

RESULTS: $\quad$ Eight pigs were anesthetized, mechanically ventilated, and equipped. Both norepinephrine and epinephrine significantly increased IVCF and decreased PPV and SVV, regardless of volemic conditions $(p<0.05)$. However, epinephrine was also able to significantly increase $\mathrm{CO}$ regardless of volemic conditions.

CONCLUSION: The present study demonstrates that intravenous administrations of norepinephrine and epinephrine increase IVCF, whatever the volemic conditions are. The concomitant decreases in PPV and SVV corroborate the fact that catecholamine administration recruits unstressed blood volume. In this regard, understanding a decrease in PPV and SVV values, after catecholamine administration, as an obvious indication of a restored volemia could be an outright misinterpretation. (J Trauma Acute Care Surg. 2014;77: 564-569. Copyright (C 2014 by Lippincott Williams \& Wilkins)

KEY WORDS: Fluid responsiveness; dynamic indices; cardiac output; venous return; catecholamines.

Vo olume status assessment, cardiac performance, and proper use of volume expansion (VE) are important procedures in the intensive care unit. ${ }^{1}$ When faced with acute circulatory failure, $\mathrm{VE}$ is one of the first therapeutic options to restore, even partially, decreased cardiac output (CO). ${ }^{2}$ In addition, although catecholamines produce various adverse effects, norepinephrine (NE) or epinephrine (EPI) may be needed to maintain mean arterial pressure (MAP). ${ }^{3}$

Indeed, the Frank-Starling law establishes a curvilinear physiologic relationship between preload and stroke volume with an expected change of the latter after VE. ${ }^{4,5}$ Much research has concentrated on the assessment of left ventricular preload dependency parameters. ${ }^{6}$ Such indices are defined as either static or dynamic markers. Static indices measure one parameter at a set point of the ventilatory cycle and do not reliably predict VE responsiveness. $^{7,8}$ Dynamic indices measure the magnitude of

Submitted: April 1, 2014, Revised: June 4, 2014, Accepted: June 6, 2014.

From the Intensive Care Unit (R.G., N.S., D.A., K.B.), Geneva University Hospitals; Geneva Medical Faculty (R.G., N.S., K.B.), University of Geneva; and Geneva Hemodynamic Research Group (R.G., N.S., K.B.), Geneva, Switzerland.

Supplemental digital content is available for this article. Direct URL citations appear in the printed text, and links to the digital files are provided in the HTML text of this article on the journal's Web site (www.jtrauma.com).

Address for reprints: Raphaël Giraud, MD, MSc, Intensive Care Unit, Geneva University Hospital, 4 rue Gabrielle Perret-Gentil, CH-1211 Geneva 14, Switzerland; email: Raphael.Giraud@hcuge.ch.

DOI: 10.1097/TA.0000000000000409 a parameter's variation during a positive-pressure ventilatory cycle and accurately predict VE responsiveness. ${ }^{9,10}$ The underlying concept is a biventricular functional assessment of the Frank-Starling curves, which permits the prediction of preload dependency. ${ }^{11}$ In this regard, the two most widely studied dynamic indices are stroke volume respiratory variation (SVV) and arterial pulse pressure respiratory variation (PPV).

However, it is not sufficient to consider the heart as the sole determinant of cardiovascular system. Indeed, equally important are the works of Guyton et al. ${ }^{12}$ emphasizing the major impact of venous return on $\mathrm{CO}$. Given that systemic circulation is a closed loop system, the mean left ventricular $\mathrm{CO}$ is equal to the systemic venous return. Interestingly, the venous system holds up to $65 \%$ of the total blood volume; with only a portion of the blood volume being available (the "effective blood volume"), which acts as a crucial factor in venous return and thus $\mathrm{CO}$.

Catecholamine vasopressors are used in the intensive care unit to maintain vital organ perfusion pressure until the resolution of shock. However, the effects of these agents on the venous side (blood pooling) have not been extensively studied during acute hypovolemia. ${ }^{13}$ Indeed, several hemodynamic studies evaluating the effects of catecholamine on blood circulation have focused on cardiac performance and arterial tone without systematically assessing the role of venous vessels as a part of the systemic circulation. In this regard, considerable confusion exists regarding the benefit of vasoconstrictors on the circulatory system during hemorrhage. Ascribing the benefit to 
merely an impact on the heart (coronary arteries) and the arterial system has become the vogue.

A more realistic conception of the positive effect of catecholamines on $\mathrm{CO}$, during hemorrhage, is to consider the role of these agents on veins (enhancement of venous return via venoconstriction) rather than on ventriculoarterial coupling. ${ }^{12}$ In other words, does catecholamines infusion able to increase cardiac preload in hypovolemic subjects? Accordingly, the present study was designed to clarify whether intravenous (IV) administrations of NE and EPI impact PPV and SVV through an effect on venous return.

\section{MATERIALS AND METHODS}

Approval from the Ethics Committee for Animal Research of the Canton of Geneva (Switzerland) was obtained before initiating the study. Animal handling was performed according to the Guide for the Care and Use of Laboratory Animals (Institute of Laboratory Animal Resources, 1996).

The animals were premedicated with ketamine $(15 \mathrm{mg} / \mathrm{kg})$ and midazolam $(0.3 \mathrm{mg} / \mathrm{kg})$ and anesthetized by propofol injection $(2 \mathrm{mg} / \mathrm{kg})$ followed by continuous propofol infusion $(100 \mu \mathrm{g} / \mathrm{kg} / \mathrm{min})$ and fentanyl $(10 \mu \mathrm{g} / \mathrm{kg} / \mathrm{h})$. After tracheal intubation, the pigs were mechanically ventilated (Servo ventilator $900 \mathrm{C}$-Siemens-Elema AB, Solna, Sweden) using a contant tidal volume $(10 \mathrm{ml} / \mathrm{kg})$, a respiratory rate set to maintain end-expiratory $\mathrm{CO}_{2}$ partial pressure within a normal range, an end-expiratory pressure of $5 \mathrm{~cm} \mathrm{H}_{2} \mathrm{O}$, and a fixed inspiratoryexpiratory time ratio (1:2). A lactated Ringer's infusion $5 \mathrm{~m} / \mathrm{kg}$ per hour was administered to account for basal fluid loss. All of the animals were equipped with central venous catheters in the internal jugular vein and femoral artery catheters (Pulsiocath, Pulsion Medical Systems AG, Munich, Germany).

After median sternotomy and longitudinal pericardiotomy, ultrasound transit time flow probes were placed around the aortic root and inferior vena cava (IVC) (14 mm A series, Transonic System, Ithaca, NY). The IVC probe was positioned around the vessel just below the junction of the right atrium. We used a probe system with a probe body and a space to receive in a secure, detachable fashion the compliant venous vessel. A 3-0 Prolene suture was placed around the IVC for intermittent vascular occlusion analysis. As a final point, an apical stab using a needle was performed in the left ventricle before it was catheterized with a 7 Fr high-fidelity conductance pressure-volume (P-V) catheter (Scisense Inc., London, Ontario, Canada). The catheter was advanced along the long axis of the left ventricle. In two animals, the conductance P-V catheter was positioned via the right carotid artery and not the apex. The correct position was determined by online visualization of the P-V loops and by the ability to modify the P-V loops when altering preload through occlusion of the IVC. The pericardium was partially closed and suspended as a pericardial cradle. Thoracic drains were externally inserted into the pleural space and positioned under water to prevent air from entering the thorax. Pleural pressure was recorded with an air-filled balloon placed in the mediastinal pleural space before closing the chest (Marquat, Ref C48 Sde Guenard, Boissy-St-Leger, France).

A 10-minute stabilization period was observed, during which VE was administered to obtain a minimal $5 \mathrm{~mm} \mathrm{Hg}$ of central venous pressure (CVP) and a PPV under 10\%. Normocapnia was assessed by end-tidal $\mathrm{CO}_{2}$ partial pressure $\left(\mathrm{PEtCO}_{2}\right)$ with targets between $35 \mathrm{~mm} \mathrm{Hg}$ and $45 \mathrm{~mm} \mathrm{Hg}$ (capnometry monitor, Dräger Medical, Telford, PA) and further confirmed by arterial blood gas analysis.

\section{Data Acquisition and Analysis}

A standard 3-lead electrocardiogram (ECG) was continuously displayed on a monitor (Hewlett-Packard, Andover, MA) with a heart rate digital readout by transcutaneous electrodes. Pin ECG electrodes were inserted into the chest wall skin. Arterial blood pressure and ECG signals were continuously recorded and stored at a sampling rate of $500 \mathrm{~Hz}$. We collected continuous measurements for CVP, invasive arterial blood pressure, airway pressure, and pulse pressure. Regarding the flows, IVC flow (IVCF), $\mathrm{CO}\left(\mathrm{CO}_{\mathrm{AF}}\right)$, and SVV were assessed using ultrasound transit time flow probes (Transonic System).

In four animals, P-V loops data were measured and analyzed using a pressure volume system (ADVantage system, Transonic Scisense, Inc.). We calibrated the ADVantage system as described in the literature, ${ }^{14}$ measuring the concomitant stroke volume and using the aortic flow probe for calibration. All of the pressures, P-V loops signals, and flow signals were recorded using a multichannel recording system (MP 150, Biopac System, Santa Barbara, CA) and were assessed using a Starter system for PC/Windows (AcqKnowledge software, Biopac Systems). The data acquired online were stored on a laptop computer for subsequent analysis.

The circulatory data were averaged over five breaths; systolic and diastolic pressures were measured on a beat-by-beat basis to calculate pulse pressure and stroke volume (aortic flow probe). The PPV was defined as the difference between the maximal and minimal values of pulse pressure divided by the average of the pulse pressure during a respiratory cycle. The SVV was defined as the difference between the maximal and minimal values of stroke volume divided by the average of the stroke volume during a respiratory cycle. The PPV and SVV values used for statistical analysis were the average measurements of five consecutive respiratory cycles during the defined circulatory state. ${ }^{15,16}$

\section{Experimental Protocol}

A 15-minute stabilization period was observed after surgical preparation in the present experimentation (Fig. 1). Under steadystate anesthesia and normal MAP, circulatory and respiratory variables were recorded. A first set of normovolemia (B, baseline)

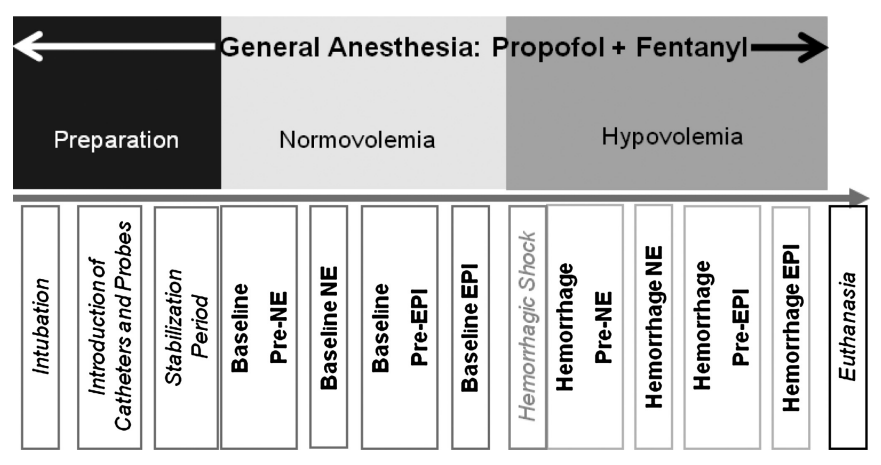

Figure 1. Swine model hemorrhagic shock protocol. 
TABLE 1. Hemodynamic Values During the Protocol

\begin{tabular}{|c|c|c|c|c|}
\hline & Pre-NE & NE & Pre-EPI & EPI \\
\hline \multicolumn{5}{|l|}{ MAP, mm Hg } \\
\hline Baseline & 70.8 (14.9) & $88.8(18.8)^{*}$ & 71.9 (17.6) & $81.3(16.3)^{* *}$ \\
\hline Hemorrhage & $46.1(8.9) \dagger$ & $70.1(16.9)^{* \dagger}$ & $46.5(8.8) \dagger$ & $67.4(18.3)^{* * \dagger}$ \\
\hline \multicolumn{5}{|l|}{$\mathrm{HR}$, beats/min } \\
\hline Baseline & $94.6(24.7)$ & $124.6(44.3)^{*}$ & $96.9(24.3)$ & $146.8(33)^{* *}$ \\
\hline Hemorrhage & $117.6(23.8) \dagger$ & $139.5(26.3)^{*}$ & $113.3(15.8)$ & $151.0(21.9)^{* *}$ \\
\hline Hemorrhage & $10.1(1.3) \dagger$ & $9.1(4.3)$ & $9.9(2.3) \dagger$ & $10.5(2.4)$ \\
\hline \multicolumn{5}{|l|}{ PPV, \% } \\
\hline Baseline & $6.9(2.1)$ & $6.1(1.7)$ & $7.8(2)$ & $6.6(1.5)$ \\
\hline Hemorrhage & $16.4(4.8) \dagger$ & $11.8(4.2)^{* \dagger}$ & $14.3(4.1) \dagger$ & $11.5(4.9)^{* * \dagger}$ \\
\hline \multicolumn{5}{|l|}{ SVV, \% } \\
\hline Baseline & $11.5 .2(3.7)$ & $9.7(1.6)$ & $11.0(5.3)$ & $9.9(1.9)$ \\
\hline Hemorrhage & $0.71(0.25) \dagger$ & $0.97(0.42)^{* \dagger}$ & $0.71(0.19) \dagger$ & $1.09(0.29)^{* * \dagger}$ \\
\hline \multicolumn{5}{|l|}{$\mathrm{CO}, \mathrm{L} / \mathrm{min}$} \\
\hline Baseline & $1.6(0.28)$ & $2.01(0.25)^{*}$ & $1.68(0.37)$ & $2.49(0.53)^{* *}$ \\
\hline Hemorrhage & $1.42(0.91)$ & $1.86(0.75)^{*}$ & $1.53(0.76)$ & $1.94(0.77)^{* *}$ \\
\hline \multicolumn{5}{|l|}{$\mathrm{SV}, \mathrm{mL}$} \\
\hline Baseline & $19.0(2.4)$ & $18.8(1.9)$ & $19.5(2.4)$ & $18.6(2.1)$ \\
\hline Hemorrhage & $13.3(3.8) \dagger$ & $14.4(2.5) \dagger$ & $14.2(3.6) \dagger$ & $13.6(2.5) \dagger$ \\
\hline
\end{tabular}

measurements was performed before NE administration (B pre-NE). A continuous NE infusion was subsequently titrated to obtain a $15 \%$ increase in MAP with a new set of measurements (B-NE). The NE infusion was interrupted, and after a steady state, measurements were again obtained before EPI administration (B pre-EPI). Finally, a continuous EPI infusion was titrated to achieve a $15 \%$ increase in MAP before performing the final measurements in the normovolemic state (B-EPI).

Hemorrhagic shock $(\mathrm{H})$ was subsequently produced by removing blood from the femoral artery catheter to obtain a MAP less than $60 \mathrm{~mm} \mathrm{Hg}$ in 5 minutes to 10 minutes. Because in the majority of the cases, the arterial pressure was partially restored because of the spleen's ability to sequester red blood cells and to induce subsequent autotransfusion, additional withdrawal of blood was necessary. This procedure usually entailed removing $30 \%$ of the total blood volume $(30 \mathrm{~mL} / \mathrm{kg}$ representing $750[30] \mathrm{mL})$ within 30 minutes. The same measurements were obtained during the present hemorrhagic state (H pre-NE, H-NE, H preEPI, and H-EPI). All of the measurements, under each circulatory state $\mathrm{B}$ or $\mathrm{H}$, before and after the administration of medications, were obtained after a 10-minute stabilization period. The animals were killed by euthanasia using a lethal injection of potassium chloride

\section{Statistical Analysis}

The results are presented as mean (SD); each animal served as its own control using paired data. All analyzed data were numerical and continuous as follows: PPV (\%), SVV (\%), IVCF (L/min), MAP ( $\mathrm{mm} \mathrm{Hg}$ ), CVP ( $\mathrm{mm} \mathrm{Hg}$ ), systolic ejection volume $(\mathrm{SV})\left(\mathrm{mL} / \mathrm{min} / \mathrm{m}^{2}\right)$, and heart rate (HR) (beats $\left./ \mathrm{min}\right)$. These values are presented in box-and-whisker plots. Data were compared between normovolemic baseline conditions (B) and hemorrhagic shock $(\mathrm{H})$ both before and after administrations of $\mathrm{NE}$ and EPI using a nonparametric Wilcoxon test. Analysis was performed using SPSS software 17.0 (SPSS Inc., Chicago, IL), and $p<0.05$ was considered to be statistically significant.

\section{RESULTS}

The study included eight domestic pigs (mean [SD] weight range, 25 [2] kg). The collected hemodynamic values at baseline and hemorrhagic states are summarized in Table 1. Each individual parameter is shown in Figures 2 to 5 , according to each protocol step. Because tidal volume, ${ }^{16-18}$ respiratory rate, ${ }^{19}$ thoracic compliance, ${ }^{20,21}$ and positive end-expiratory pressure levels ${ }^{22,23}$ all affect PPV and SVV, these parameters were kept within strict ranges throughout the entire study.

Overall, the hypovolemic state induced significant decreases in MAP, IVCF, CVP, and SV with concomitant increases in PPV, SVV, and HR ( $p<0.05$, Table 1, Figs. 2-5). NE and EPI significantly increased MAP, HR, IVCF, and CO $(p<$ 0.05 ) whatever the circulatory condition is (Table 1, Figs. 2 and 5). The PPV and SVV were significantly diminished by the 


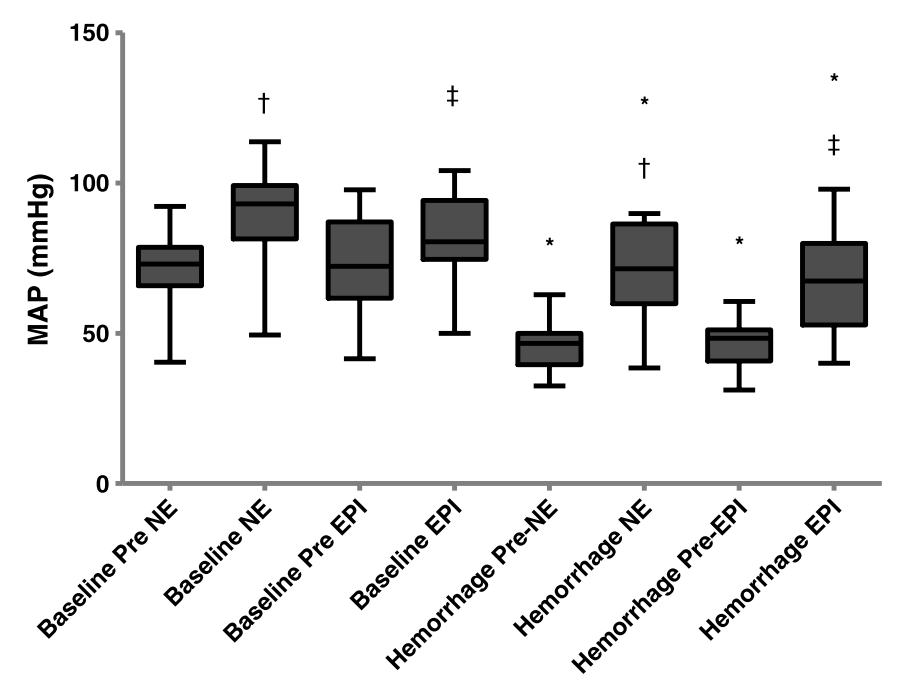

Figure 2. Box plot representing MAP according to study protocol. *Statistically significant between baseline and hemorrhage $(p<0.05)$. † Statistically significant between pre-NE and NE $(p<0.05)$. $\$$ Statistically significant between pre-EPI and $\operatorname{EPI}(p<0.05)$.

administrations of NE and EPI during normovolemia and hemorrhagic state $(p<0.05$, Table 1, Figs. 3 and 4$)$.

In the four animals where P-V loops data were analyzed, the data showed that NE increased left ventricular end-diastolic volume in the absence of an increase in enddiastolic pressure (see Figure, Supplemental Digital Content 1, http://links.lww.com/TA/A465).

\section{DISCUSSION}

Our study results on normovolemic and hypovolemic anesthetized pigs allow several important assertions. Both PPV

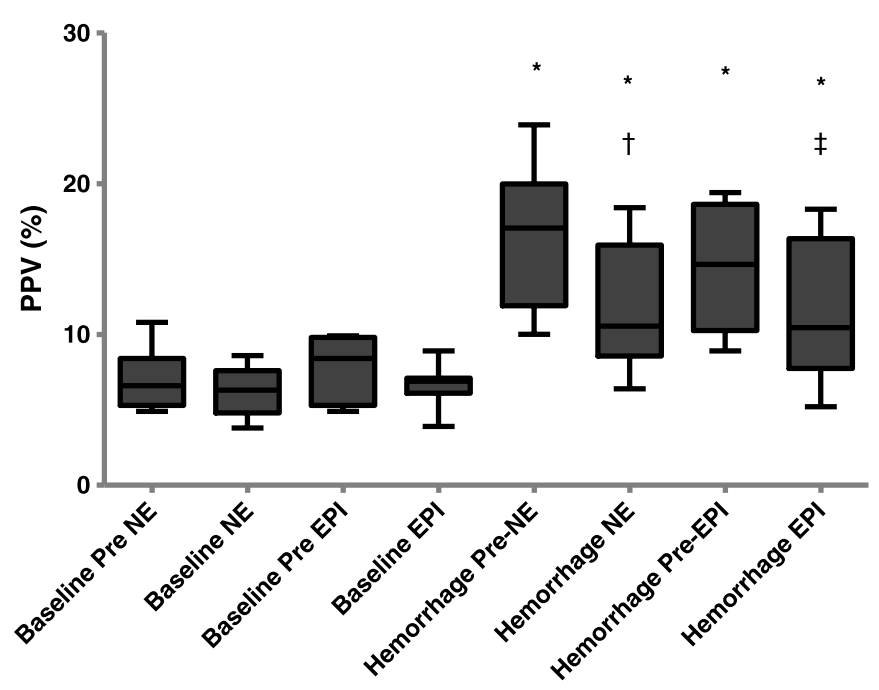

Figure 3. Box plot representing PPV according to study protocol. *Statistically significant between baseline and hemorrhage $(p<0.05)$. $\uparrow$ Statistically significant between pre-NE and NE $(p<0.05)$. $\$$ Statistically significant between pre-EPI and EPI $(p<0.05)$.

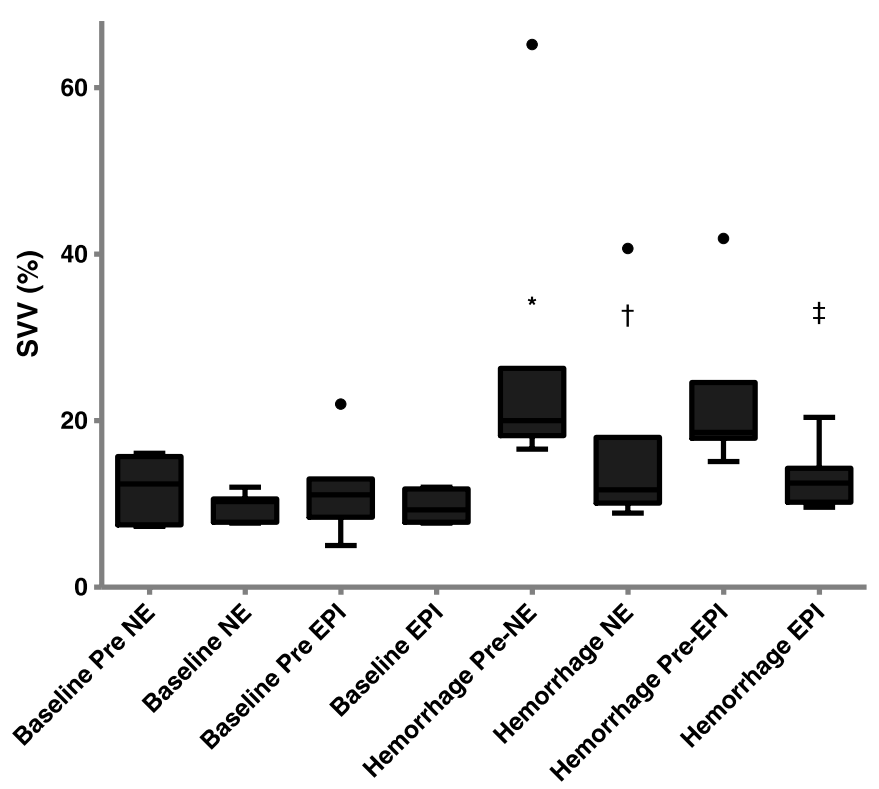

Figure 4. Box plot representing SVV according to study protocol. *Statistically significant between baseline and hemorrhage $(p<0.05)$. $\uparrow$ Statistically significant between pre-NE and NE $(p<0.05)$. $\$$ Statistically significant between pre-EPI and EPI $(p<0.05)$.

and SVV significantly increase after hemorrhagic shock ${ }^{24}$ and diminish significantly under NE and EPI perfusions. Of significance, the present study demonstrates that decreases in PPV and SVV values after catecholamine infusions are related to an autotransfusion effect as highlighted by the increase in IVCF.

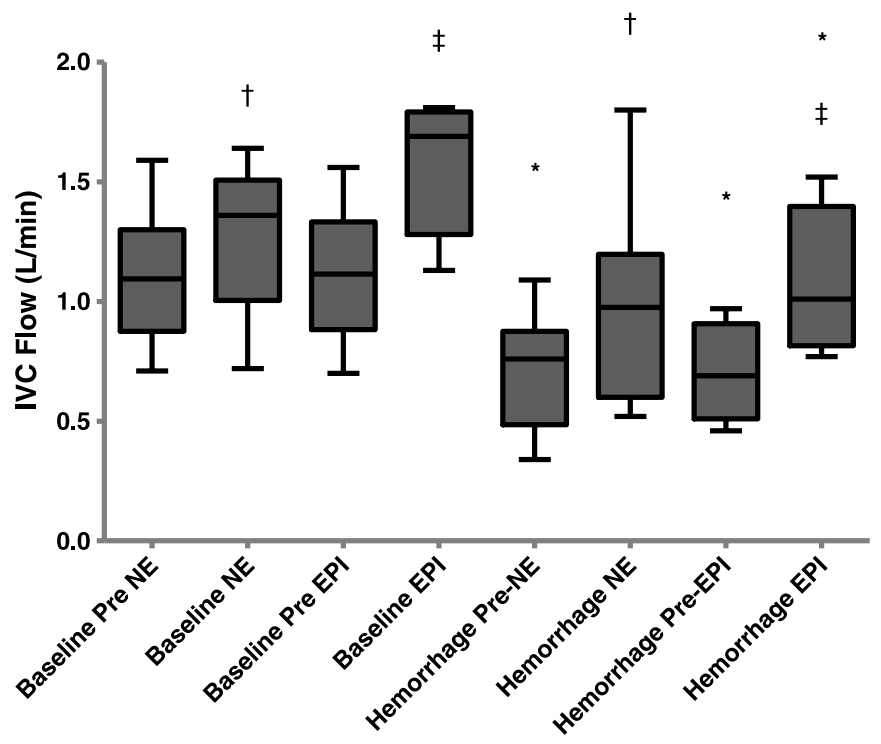

Figure 5. Box plot representing IVCF according to study protocol. *Statistically significant between baseline and hemorrhage $(p<0.05)$. $\uparrow$ Statistically significant between pre-NE and NE $(p<0.05)$. \$Statistically significant between pre-EPI and EPI $(p<0.05)$. 
EPI is a natural hormone, which is secreted and produced by the adrenal gland with direct $\alpha$ - and $\beta$-agonist action. EPI has dose-dependent $\alpha_{1}, \alpha_{2}, \beta_{1}$, and $\beta_{2}$ effects with $\beta_{2}$ vasodilation at minor doses and potent vasoconstriction through $\alpha$ receptor stimulation at higher doses. The heterogeneous receptor distribution makes its action mechanism analysis complex. $\mathrm{NE}$, which is an EPI-precursor that differs only by a methyl group on the terminal amine function, has primarily $\alpha_{1}$ and $\alpha_{2}$ effects, minor $\beta_{1}$, and no $\beta_{2}$. Catecholamines increase venous return during hemorrhagic shock by transferring blood from the unstressed to the stressed compartment. The mechanism by which the present blood shift is achieved would be a decrease in venous compliance or increase in elastance and venous conductance. By harvesting blood from the splanchnic territory, for example, catecholamines could increase the venous return and stroke volume, thereby diminishing preload dependency and dynamic indices. ${ }^{25,26}$

In this regard, both PPV and SVV, which are valid indices of fluid depletion and fluid responsiveness, ${ }^{24}$ diminished significantly under NE and EPI perfusions. The present hemodynamic changes were related to changes in venous return, as we demonstrated that IVCF, which diminishes during hypovolemia, increased following catecholamine infusions. These results are supported by clinical practice observation as VE associated with catecholamines improves cardiovascular function in unstable patients. As previously stated, NE and EPI have major impacts on vascular redistribution. Fifty years ago, Guyton et al. ${ }^{12,27}$ demonstrated that EPI increased CO primarily through increased venous return by shifting blood volume from the unstressed to the stressed compartment. ${ }^{28-30}$ Our study confirms previously published data, ${ }^{31-33}$ stating that both EPI and NE increase venous return by increasing IVCF. If our results did not show significant CVP changes after EPI and NE infusions, we believe that the reason is because we measured intravascular CVP, which was at the same time decreased by better relaxation of the right atrium (inotropic effect) and increased via a greater IVCF. ${ }^{34}$ Catecholamines therefore increase venous return by a combination of lower venous compliance (unstressed-stressed blood shift) and lower transmural cardiac pressures (lusitropic effect). Indeed, a series of animal studies have estimated the unstressed venous blood volume to be $70 \%$ to $75 \%$ of the total blood volume. ${ }^{35,36}$ Whether the spleen plays an important role in vascular redistribution has not yet been established. The impact of EPI perfusions on the circulatory function could be also related to the fact that these drugs are potent inotropes, which may increase $\mathrm{CO}$ through an increase in contractility or through an increase in HR.

Another way for vasopressors to influence PPV is by directly reducing arterial vascular compliance (increase in PPV). Thus, because catecholamines have a direct effect on both regional vascular capacitance (i.e., venous return) and arterial tone, these hormone agents may at the same time decrease and increase PPV value. The resulting merged PPV value in this setting may explain why the diagnostic accuracy of PPV for the prediction of fluid responsiveness could be influenced in some cases. ${ }^{37}$ However, an experimental study performed on dogs demonstrated that PPV decreased during an NE perfusion, despite increased vascular tone. ${ }^{25}$ As mentioned earlier, the present result is related to the fact that vasopressors also diminish PPV by increasing venous return. Without doubt, our study confirms the hypothesis that vasopressors, such as NE and EPI, increase venous return and ventricular preload (Fig. 5) while diminishing both PPV and SVV.

The present animal study is of clinical value because it also emphasizes that under experimental conditions, intramural CVP modifications are not correlated to $\mathrm{CO}$ variations. Moreover, this same present study underlines that NE and EPI control an autotransfusion effect. In this regard, physicians should consider the present physiologic concept when interpreting PPV and SVV values in patients who receive vasopressors because usual thresholds $(13 \%)^{9}$ could be too high in this setting. ${ }^{25,37,38}$ For instance, a truly hypovolemic patient may decrease PPV and SVV with the administration of NE; the physician could either inaccurately pursue vasopressor administration or interpret PPV and SVV decreases as an autotransfusion from unstressed to stressed blood volume, thus confirming the need for VE to restore unstressed blood volume. Giving fluids to these types of patients even if the PPV value is less than $13 \%$ would decrease vasopressor IV doses. Of importance is the present paradigm shift, which underlines this potential misinterpretation that may occur if physicians understand a decrease in the PPV and SVV values after catecholamine administration as a signal of normovolemia. Indeed, the present circulatory state is defined as a relative normovolemia.

The authors acknowledge three weak methodological points in the present study. First, hemorrhagic shock produced in our animal study led to a restrained hypovolemia that induced a moderate increase in the PPV (low signal). In this sense, we may speculate that the impact of catecholamine on the diminution of PPV values could have been more impressive in the case where hypovolemia was more severe. Second, in our protocol, we performed consecutive blood removal to stabilize MAP at $60 \mathrm{~mm} \mathrm{Hg}$. We may speculate that the impact of catecholamine on the reduction of $\Delta \mathrm{PP}$ values could have been more impressive in case of nonacute bleeding (as the recruitment of unstressed blood volume should be potentially higher). Finally, catecholamine infusions before or during hemorrhage were applied in a fixed order without randomization, and since NE and EPI produced changes in PPV and SVV, there had to be fluid shifts with a possible drift in the baseline conditions. However, there was no statistical difference between pre-drug infusion setting whatever the circulatory condition is.

\section{CONCLUSION}

The present study demonstrates that in pigs, the IV administration of NE and EPI increases both IVCF and CO whatever the circulatory state is. Of importance is the present paradigm shift that underlines that even if PPV and SVV values associated with $\mathrm{NE}$ and EPI infusions remain indicative of changes in volemia, threshold values identifying hypovolemia might be lower. Indeed, understanding the decrease in PPV and SVV values after catecholamine administration as an obvious indication of a restored volemia could be an outright misinterpretation.

\section{AUTHORSHIP}

R.G. performed the animal exprimentation, performed the statistical analysis, interpreted the date, and wrote the article. N.S. performed the animal exprimentation and wrote the article. D.A. performed the animal 
exprimentation and wrote the article. K.B. designed the study, performed the animal exprimentation, interpreted the data and wrote the article. All authors agreed with the final version of this article.

\section{DISCLOSURE}

The authors declare no conflicts of interest.

\section{REFERENCES}

1. Bendjelid K, Romand JA. Fluid responsiveness in mechanically ventilated patients: a review of indices used in intensive care. Intensive Care Med. 2003;29:352-360.

2. Rivers E, Nguyen B, Havstad S, et al. Early goal-directed therapy in the treatment of severe sepsis and septic shock. N Engl J Med. 2001; 345:1368-1377.

3. Carcillo JA, Fields AI. Clinical practice parameters for hemodynamic support of pediatric and neonatal patients in septic shock. Crit Care Med. 2002;30:1365-1378

4. Starling EH. The Linacre Lecture on the Law of the Heart. London, UK: Longmans, Green and Co; 1918.

5. Stetz CW, Miller RG, Kelly GE, Raffin TA. Reliability of the thermodilution method in the determination of cardiac output in clinical practice. Am Rev Respir Dis. 1982;126:1001-1004.

6. Coudray A, Romand JA, Treggiari M, Bendjelid K. Fluid responsiveness in spontaneously breathing patients: a review of indexes used in intensive care. Crit Care Med. 2005;33:2757-2762.

7. Hinder F, Poelaert JI, Schmidt C, Möllhoff T, Loick HM, Van Aken H. Assessment of cardiovascular volume status by transoesophageal echocardiography and dye dilution during cardiac surgery. Eur J Anaesthesiol. 1998;15:633-640.

8. Reuse C, Vincent JL, Pinsky MR. Measurements of right ventricular volumes during fluid challenge. Chest. 1990;98:1450-1454.

9. Michard F, Teboul JL. Using heart-lung interactions to assess fluid responsiveness during mechanical ventilation. Crit Care. 2000;4:282-289.

10. Michard F, Teboul JL. Predicting fluid responsiveness in ICU patients: a critical analysis of the evidence. Chest. 2002;121:2000-2008.

11. Pinsky MR. Functional hemodynamic monitoring. Intensive Care Med. 2002;28:386-388.

12. Guyton AC, Lindsey AW, Abernathy B, Richardson T. Venous return at various right atrial pressures and the normal venous return curve. $\mathrm{Am} J$ Physiol. 1957;189:609-615.

13. Berdeaux A, Edouard A. Pharmacologie des récepteurs adrénergiques et dopaminergiques: implications thérapeutiques actuelles. In: 15e Journée Internationales de Mises au Point en Anesthésie - Réanimation, 1997, PM (Ed), 1997. p. 397.

14. Clark JE, Kottam A, Motterlini R, Marber MS. Measuring left ventricular function in the normal, infarcted and CORM-3-preconditioned mouse heart using complex admittance-derived pressure volume loops. J Pharmacol Toxicol Methods. 2009;59:94-99.

15. Michard F, Boussat S, Chemla D, Anguel N, Mercat A, Lecarpentier Y, Richard C, Pinsky MR, Teboul JL. Relation between respiratory changes in arterial pulse pressure and fluid responsiveness in septic patients with acute circulatory failure. Am J Respir Crit Care Med. 2000;162:134-138.

16. Szold A, Pizov R, Segal E, Perel A. The effect of tidal volume and intravascular volume state on systolic pressure variation in ventilated dogs. Intensive Care Med. 1989;15:368-371.

17. Reuter DA, Bayerlein J, Goepfert MS, FC Weis, Kilger E, Lamm P, Goetz $\mathrm{AE}$. Influence of tidal volume on left ventricular stroke volume variation measured by pulse contour analysis in mechanically ventilated patients. Intensive Care Med. 2003;29:476-480.

18. De Backer D, Heenen S, Piagnerelli M, Koch M, Vincent JL. Pulse pressure variations to predict fluid responsiveness: influence of tidal volume. Intensive Care Med. 2005;31:517-523.
19. De Backer D, Taccone FS, Holsten R, Ibrahimi F, Vincent JL. Influence of respiratory rate on stroke volume variation in mechanically ventilated patients. Anesthesiology. 2009;110:1092-1097.

20. Pizov R, Ya'ari Y, Perel A. The arterial pressure waveform during acute ventricular failure and synchronized external chest compression. Anesth Analg. 1989;68:150-156.

21. Tournadre JP, Allaouchiche B, Cayrel V, et al. Estimation of cardiac preload changes by systolic pressure variation in pigs undergoing pneumoperitoneum. Acta Anaesthesiol Scand. 2000;44:231-235.

22. Michard F, Chemla D, Richard C, Wysocki M, Pinsky MR, Lecarpentier Y, Teboul JL. Clinical use of respiratory changes in arterial pulse pressure to monitor the hemodynamic effects of PEEP. Am J Respir Crit Care Med. 1999;159:935-939.

23. Pizov R, Cohen M, Weiss Y, Segal E, Cotev S, Perel A. Positive endexpiratory pressure-induced hemodynamic changes are reflected in the arterial pressure waveform. Crit Care Med. 1996;24:1381-1387.

24. Fujita Y, Yamamoto T, Sano I, Yoshioka N, Hinenoya H. A comparison of changes in cardiac preload variables during graded hypovolemia and hypervolemia in mechanically ventilated dogs. Anesth Analg 2004;99: 1780-1786.

25. Nouira S, Elatrous S, Dimassi S, Besbes L, Boukef R, Mohamed B, Abroug F. Effects of norepinephrine on static and dynamic preload indicators in experimental hemorrhagic shock. Crit Care Med. 2005;33: 2339-2343.

26. Pinsky MR. The dynamic interface between hemodynamic variables and autonomic tone. Crit Care Med. 2005;33:2437-2438.

27. Guyton AC, Lindsey AW, Abernathy B, Langston JB. Mechanism of the increased venous return and cardiac output caused by epinephrine. Am J Physiol. 1958;192:126-130.

28. Appleton C, Olajos M, Morkin E, Goldman S. Alpha-1 adrenergic control of the venous circulation in intact dogs. J Pharmacol Exp Ther. 1985; 233:729-734.

29. Ruffolo RR Jr. Distribution and function of peripheral alpha-adrenoceptors in the cardiovascular system. Pharmacol Biochem Behav. 1985;22:827-833.

30. Siegenthaler N, Giraud R, Bendjelid K. A semantic point of view about unstressed blood volume. Crit Care Med. 2013;41:e237-e239.

31. Caldini P, Permutt S, Waddell JA, Riley RL. Effect of epinephrine on pressure, flow, and volume relationships in the systemic circulation of dogs. Circ Res. 1974;34:606-623.

32. Imai Y, Satoh K, Taira N. Role of the peripheral vasculature in changes in venous return caused by isoproterenol, norepinephrine, and methoxamine in anesthetized dogs. Circ Res. 1978;43:553-561.

33. Peters J, Mack GW, Lister G. The importance of the peripheral circulation in critical illnesses. Intensive Care Med. 2001;27:1446-1458.

34. Valenza F, Chevallard G, Porro GA, Gattinoni L. Static and dynamic components of esophageal and central venous pressure during intraabdominal hypertension. Crit Care Med. 2007;35:1575-1581.

35. Deschamps A, Magder S. Baroreflex control of regional capacitance and blood flow distribution with or without alpha-adrenergic blockade. Am J Physiol. 1992; 263: H1755-H1763.

36. Guyton AC. Determination of cardiac output by equating venous return curves with cardiac response curves. Physiol Rev. 1955;35:123-129.

37. Cannesson M, Le Manach Y, Hofer CK, Goarin JP, Lehot JJ, Vallet B, Tavernier B. Assessing the diagnostic accuracy of pulse pressure variations for the prediction of fluid responsiveness: a "gray zone" approach. Anesthesiology. 2011; 115:231-241.

38. Renner J, Meybohm P, Hanss R, Gruenewald M, Scholz J, Bein B. Effects of norepinephrine on dynamic variables of fluid responsiveness during hemorrhage and after resuscitation in a pediatric porcine model. Paediatr Anaesth. 2009;19:688-694. 